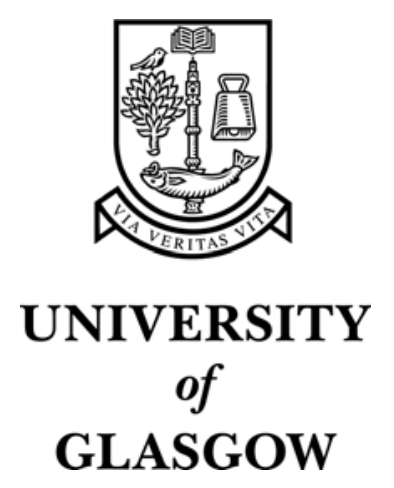

Crossan, A. and Brewster, S.A. (2006) Two-handed navigation in a haptic virtual environment. In, Conference on Human Factors in Computing Systems, 22-27 April 2006, pages pp. 676-681, Montréal, Québec, Canada.

http://eprints.gla.ac.uk/3268/ 


\section{Two-Handed Navigation in a Haptic Virtual Environment}

\author{
Andrew Crossan and Stephen \\ Brewster \\ Department of Computing Science, \\ University of Glasgow, \\ Glasgow, G12 8QQ \\ ac@dcs.gla.ac.uk \\ stephen@dcs.gla.ac.uk \\ www.dcs.gla.ac.uk/ stephen
}

\begin{abstract}
This paper describes the initial results from a study looking at a two-handed interaction paradigm for tactile navigation for blind and visually impaired users. Participants were set the task of navigating a virtual maze environment using their dominant hand to move the cursor, while receiving contextual information in the form of tactile cues presented to their non-dominant hand. Results suggest that most participants were comfortable with the two-handed style of interaction even with little training. Two sets of contextual cues were examined with information presented through static patterns or tactile flow of raised pins. The initial results of this study suggest that while both sets of cues were usable, participants performed significantly better and faster with the static cues.
\end{abstract}

\section{Keywords}

Non-visual interaction, tactile navigation, accessibility, haptics, two-handed interaction

\section{ACM Classification Keywords}

H5.2. User Interfaces: Haptic I/O, Input devices and strategies (e.g., mouse, touchscreen). 


\section{I ntroduction}

Users rely heavily on visual feedback when interacting with a computer. However, computer users with no or very little vision must rely on other modalities to access the same information. Screen readers have proved to be a successful solution for accessing the textual information required to interact with a computer. However, this information is generally accessible only in a linear manner (from the top left corner of the screen) and non-text information such as pictures and diagrams are not easily displayed in this manner. The goal of this work is to examine techniques to enable users to navigate computer interfaces and explore information non-visually in a non-linear manner. To achieve this, a two-handed focus-context interaction paradigm is adopted. Users can navigate a cursor in a 2D space and receive force-feedback by moving a device with their dominant hand. They also receive contextual information through their non-dominant hand. The contextual information in this case will be directional information displayed on a small pin array.

Similar bi-manual techniques have previously proved successful for accessing information non-visually. The Optacon [1] is one commercially available example. Visually impaired users could access printed material by moving a camera over a page with one hand while receiving a vibrotactile representation of the image under the camera presented to the other hand. Recently, Wall and Brewster [6] developed a system for browsing a bar chart with a graphics tablet and stylus for navigation. The fixed frame of reference offered by the tablet allowed users to employ their proprioceptive sense to maintain an idea of where they were within the environment. In the non-dominant hand a direct tactile analogue of the graphics was presented to the users' finger tips allowing them to browse the data through a small tactile window centred around the current cursor position.

Tactile cueing has previously been studied, for example, by van Erp and van Veen [5], who use vibrotactile cues spatially distributed around a user's torso presented through a tactile vest. Here, they use tactile patterns to indicate to astronauts their orientation with respect to the International Space Station. A recent study conducted by Martin et al. [4] examines the discriminability of different forms of cues presented to a user's fingertip through a raised pin tactile array. They examine the success of presenting a set of 8 different directional messages through different patterns of tactile cue. Different forms of static, dynamic, and 'blinking' (cycling between the pattern and an empty array) patterns are studied with the best performance noted with the static cues. For this experiment a selection of two of the sets of cues developed by Martin et al. [4] are examined. The goal of this study is to test performance in a more complex navigation task when users must integrate cues presented to different hands to navigate a virtual environment. These cues are presented using force feedback and tactile feedback.

\section{The Maze Environment}

The task chosen for the study was for the user to navigate a maze using only their haptic sense. A maze environment was chosen as it provides a constrained environment with a clearly defined goal. The user must navigate from the start position to the exit. We can therefore define an ideal path that allows an easily measurable error from this path. Also, the difficulty of a maze can be altered by adding or removing junctions. 
A visual representation of one maze used in the study is shown in figure 1 . All of the mazes for this study were similarly set in a $12 \times 12$ grid of squares.

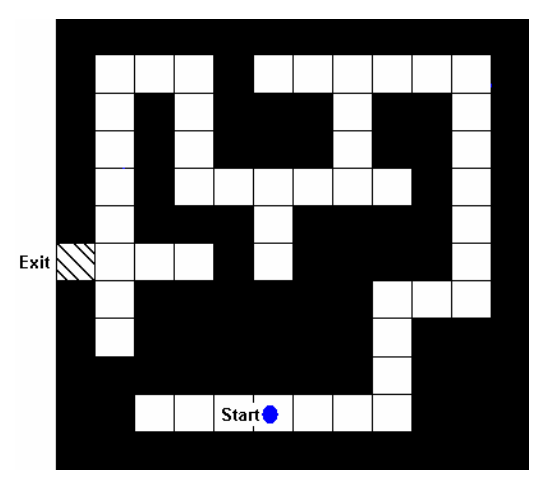

figure 1: An example maze used in the study. The white areas represent corridors and the black areas walls. The start and end positions of the maze are marked. Lines delineating the maze squares are shown visually here across the corridors.

Navigation

To navigate the maze, the user interacts with a PHANTOM OMNI force-feedback device (from SensAble Technologies) using their dominant hand. This device offers high fidelity feedback while still being relatively cheap. The device has a small wrist movement sized workspace $(160 \mathrm{~mm} \times 120 \mathrm{~mm} \times 70 \mathrm{~mm})$ and is relatively easy to overpower, which are both important safety concerns when the device arm can move independently and users cannot see the arm. Although this device allows 3D interactions, for the maze environment users are constrained by the device to 2D interactions in the vertical plane only. Users are always constrained to the corridors of the maze and can feel the maze walls (represented as stiff springs).
Tactile Cues

An initial version of the maze attempted to present a direct tactile representation of a small area around the user's cursor (described in [3]) using a VirTouch VTPlayer tactile mouse. Here, the area of the maze around the user was displayed through a tactile pin array (4 vertical $x 8$ horizontal) pins with pin-up representing a wall and pin-down a corridor (like a tactile map). Each pin represented one square on the maze centred around the user's cursor position. A pilot study with four blind participants was conducted to test the usability of this system as a navigation aid, the results of which suggested that this was an unsuccessful method of presenting the information. Al four users found the amount and the complexity of the information confusing. Each pin on the display represented a piece of information and the large number and high density of pins as well as their rapidly changing state as the user moved made the task too difficult. One user succinctly summed up the sensation as 'a tactile mess'.

It was therefore important to develop a navigation technique that reduced the complexity of information presented to the user's non-dominant hand. Instead of a visual analogy, coded tactile representations (Tactons [2]) were developed to aid user navigation. The information presented to the user was reduced to four tactile cues that provided the user the direction to the maze exit. These cues (presented on a $4 \times 4$ raised pin display) indicated to users that to get to the exit, they must move up, down, left or right. Two forms of these cues were developed: static and dynamic.

Static cues form a tactile pattern on the display with the pattern indicating the direction to move in. The 
four patterns are chosen to be similar to those developed in [4]. These patterns are shown in figure 2. Each pattern is represented by a line of raised pins on the display. The position and orientation of the pins indicates the direction the user must move in to reach the exit. If the raised pins are felt at the top of the display, the user must move up, and similarly for the other three directions. The pattern displayed remains the same until the user is required to change direction.

\begin{tabular}{|c|c|c|c|}
\hline $\begin{array}{llll}1 & 0 & 1 & 0 \\
0 & 0 & 0 & 0 \\
0 & 0 & 0 & 0 \\
0 & 0 & 0 & 0\end{array}$ & $\begin{array}{llll}0 & 0 & 0 & 0 \\
0 & 0 & 0 & 0 \\
0 & 0 & 0 & 0 \\
0 & 1 & 0 & 0\end{array}$ & $\begin{array}{llll}- & 0 & 0 & 0 \\
- & 0 & 0 & 0 \\
- & 0 & 0 & 0 \\
- & 0 & 0 & 0\end{array}$ & $\begin{array}{llll}0 & 0 & 0 & -1 \\
0 & 0 & 0 & 0 \\
0 & 0 & 0 & 0 \\
0 & 0 & 0 & 0\end{array}$ \\
\hline UP & Down & LEFT & RIGHT \\
\hline \multicolumn{4}{|c|}{ Pin Up $\bullet$} \\
\hline
\end{tabular}

figure 2: The four static cues used for the study.

The second set of cues (dynamic cues) use tactile flow to indicate direction. These offer the potential to be more expressive than the static cues as the rate of change of pins and changing patterns can now be altered to provide more information to the user.

However, they may also be more difficult for the user to interpret [4]. A series of patterns is played to the user for each of the 4 dynamic cue messages. These patterns are shown in figure 3. For each direction message, the user is played a series of five patterns where the direction of flow of the raised pins indicates the direction in which the user must move to reach the goal. In each case, the first and final patterns are left with no raised pins to allow the user to more easily separate the cues. Unlike in the static condition, the state of the display is constantly changing even when the user remains stationary. In the event of a required change in direction, the appropriate tactile cue is played from the start (always starting with an empty array). The rate of change of the display was chosen empirically at $100 \mathrm{~ms}$ per update.

\begin{tabular}{|c|c|c|c|c|c|c|}
\hline UP & 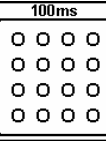 & 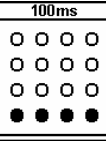 & 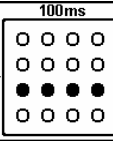 & 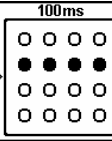 & 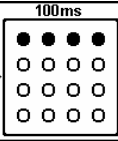 & 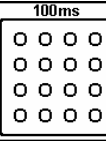 \\
\hline DOWN & $\begin{array}{llll} & 0 & 0 & 0 \\
0 & 0 & 0 & 0 \\
0 & 0 & 0 & 0 \\
0 & 0 & 0 & 0 \\
\end{array}$ & 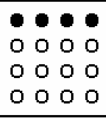 & \begin{tabular}{|llll}
0 & 0 & 0 & 0 \\
0 & 0 & 0 & 0 \\
0 & 0 & 0 & 0 \\
0 & 0 & 0 & 0 \\
\end{tabular} & $\begin{array}{|llll|}0 & 0 & 0 & 0 \\
0 & 0 & 0 & 0 \\
0 & 0 & 0 & 0 \\
0 & 0 & 0 & 0 \\
\end{array}$ & \begin{tabular}{|lllll}
0 & 0 & 0 & 0 \\
0 & 0 & 0 & 0 \\
0 & 0 & 0 & 0 \\
0 & 0 & 0 & 0 \\
\end{tabular} & \begin{tabular}{|llll}
0 & 0 & 0 & 0 \\
0 & 0 & 0 & 0 \\
0 & 0 & 0 & 0 \\
0 & 0 & 0 & 0 \\
\end{tabular} \\
\hline LEFT & $\begin{array}{llll}0 & 0 & 0 & 0 \\
0 & 0 & 0 & 0 \\
0 & 0 & 0 & 0 \\
0 & 0 & 0 & 0 \\
\end{array}$ & $\begin{array}{llll}0 & 0 & 0 & : \\
0 & 0 & 0 & 0 \\
0 & 0 & 0 & 0 \\
0 & 0 & 0 & :\end{array}$ & \begin{tabular}{|llll}
$\circ$ & 0 & $\bullet$ & 0 \\
0 & 0 & $\bullet$ & 0 \\
$\circ$ & 0 & 0 & 0 \\
0 & 0 & $\bullet$ & 0 \\
\end{tabular} & \begin{tabular}{|llll}
$\circ$ & 0 & 0 & 0 \\
$\circ$ & 0 & 0 & 0 \\
$\circ$ & 0 & 0 & 0 \\
$\circ$ & $\bullet$ & 0 & 0 \\
\end{tabular} & $\begin{array}{llll}- & 0 & 0 & 0 \\
0 & 0 & 0 & 0 \\
0 & 0 & 0 & 0 \\
0 & 0 & 0 & 0 \\
\end{array}$ & \begin{tabular}{|llll}
0 & 0 & 0 & 0 \\
0 & 0 & 0 & 0 \\
0 & 0 & 0 & 0 \\
0 & 0 & 0 & 0 \\
\end{tabular} \\
\hline RIGHT & $\begin{array}{llll}0 & 0 & 0 & 0 \\
0 & 0 & 0 & 0 \\
0 & 0 & 0 & 0 \\
0 & 0 & 0 & 0\end{array}$ & 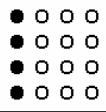 & $\begin{array}{llll}\circ & 0 & 0 & 0 \\
0 & 0 & 0 & 0 \\
\circ & 0 & 0 & 0 \\
0 & \bullet & 0 & 0 \\
\end{array}$ & $\begin{array}{llll}0 & 0 & 0 \\
0 & 0 & 0 \\
0 & 0 & 0 \\
0 & 0 & 0 \\
0 & 0 & 0\end{array}$ & {$\left[\begin{array}{llll}0 & 0 & 0 & : \\
0 & 0 & 0 & : \\
0 & 0 & 0 & : \\
0 & 0 & 0 & \bullet\end{array}\right.$} & $\begin{array}{llll}0 & 0 & 0 & 0 \\
0 & 0 & 0 & 0 \\
0 & 0 & 0 & 0 \\
0 & 0 & 0 & 0\end{array}$ \\
\hline \multicolumn{7}{|c|}{$\mathrm{J} \bullet$} \\
\hline
\end{tabular}

figure 3: The four dynamic tactile cues used in the study. Each row represents a series of patterns played to the user (at a rate of 10 patterns a second) to indicate a direction.

\section{Experiment}

An experiment was conducted to examine the performance of visually impaired users on this system.

\section{Methodology}

There were two conditions for the experiment: static or dynamic cues. Users were set the task to navigate to the maze exit within 50 seconds with audio cues alerting users of the amount of time that they had remaining. A within-subjects design was used such that all participants performed both conditions in a counterbalanced order. Ten participants from the Royal National College for the Blind in Hereford (UK) took 
part in the study. Participants had a range of visual impairments from congenital blindness to some residual sight remaining. In all instances, instructions for the experiment were provided verbally. To ensure the complexity of the mazes was kept constant, the mazes in one condition were mirror images of the mazes in the other condition. There were 8 mazes in each condition making 160 trials in total during the experiment.

\section{Training}

At the start of the experimental session, the user was initially presented with a physical representation of a maze (built using LEGO blocks) and the equivalent computer representation using the PHANTOM only. This allowed the users to familiarize themselves with both the concept of the maze environment and the PHANTOM device by exploring both simultaneously. Before each condition users were familiarized with the 4 appropriate cues for the condition, then presented with three mazes using the appropriate tactile cues for navigation and asked to navigate to the goal.

\section{Hypotheses}

1. Participants will successfully complete more mazes within the given time frame in the static condition

2. Participants will perform faster in the static condition

3. Participants will travel less distance when completing a maze in the static condition. This will be measured by examining the excess path length as proportion of the ideal path length (both measured in squares traversed) for successfully completed mazes only.
Results

Data collected from 15 mazes were unusable in the analysis. The most common reason for this was the user applying excessive force and overpowering the device, and thus pushing through walls. When data was discarded from a maze, the mirror image data from the other condition for the same participant was also discarded to maintain data that could be compared over both conditions. There were a total of 65 paired mazes from each condition that provided data that were usable in the results. Participants in total completed 58 in the static condition compared to 47 in the dynamic condition. The paired difference in performance for each participant was analysed using a non-parametric Wilcoxon Signed Rank Test and a significant difference was found ( $\mathrm{W}=33.0, \mathrm{p}<0.05$ ) supporting hypothesis 1 .

Data for hypothesis 2 were analysed using a paired Ttest. Times for unfinished mazes were set at the timeout value of 50 seconds for the analysis. Mean time in seconds for each maze was 23.1 (stdev $=9.12$ ) in the static condition compared to 34.4 (stdev = 10.26) in the dynamic condition. This difference is significant $\left(T_{9}=2.62, p<0.03\right)$ supporting hypothesis 2 .

An Anderson-Darling test demonstrated that the path length data was not normally distributed. A nonparametric Wilcoxon Signed Rank Test on the difference in path length for each user was therefore used to test significance. When completing a maze, the mean excess number of squares traversed was 0.93 (stdev = 0.86 ) times the ideal path length in the static condition compared to 1.09 (stdev $=1.03$ ) times the ideal path length in the dynamic condition. This difference is not 
significant ( $W=31.0, p=0.76)$. We cannot therefore support hypothesis 3 with the current data.

\section{Observational results}

Of the 10 participants that took part in the study, all but one expressed a preference for the static cues over the dynamic cues. One potential reason for this could be due to the fact that all had previous experience with Braille (which is another static coded tactile representation) and the sensation of moving pins conveying information is a novel experience. Two users had trouble with the two-handed nature of the task. One user preferred to concentrate on the feedback from the PHANTOM and only used the tactile array while holding the PHANTOM stationary. The other required convincing to keep a hand on the tactile array and stated they rarely used the feedback from it.

\section{Discussion and Conclusions}

The above results suggest that most users could successfully use the two-handed technique to navigate the maze environment. Users performed better and faster using the static patterns as was suggested by [4]. However, even in the dynamic condition, users still completed over $70 \%$ of the mazes successfully with little training. There was no difference in path length detected, although this is mainly due to the high level of variability in the data. The data gathered in this study will now be further analysed to look for differences between conditions to examine, for example, reaction times to change direction at junctions in the different conditions or differences in the success of perceiving each cue within a condition. One area of future work will be to encode more information in either moving or blinking patterns. For example, the distance to the next turning position could be encoded in the rate of change of the pattern.

This study used a virtual maze environment but techniques here can be generalised. Future work will involve using combinations of force and tactile feedback with the addition of auditory feedback in different computer environments to allow user to browse and navigate data non-visually.

\section{Acknowledgements}

This work was funded by the MICOLE project through the European Commission Framework 6.

\section{References}

[1] Bliss, J.C., et al., Optical-to-Tactile image conversion for the blind. IEEE Transactions on ManMachine Systems MMS-11, (1970), 78-83.

[2] Brown, L.M., Brewster, S.A., and Purchase, H.C. A First Investigation into the Effectiveness of Tactons. Proc. World Haptics, (2005), 167-176.

[3] Crossan, A. and Brewster, S. MICOLE - Inclusive Interaction for Data Creation, Visualisation and Collaboration. Hands on Haptics Workshop, CHI 2005, (2005).

[4] Martin, B., Pecci, I., and Pietrzak, T., Static and dynamic tactile directional cues experiment with VTPlayer mouse. Technical Report, Université Paul Verlaine- Metz (2006)

5] van Erp, J.B.F. and van Veen, H.A.H.C. A Multipurpose Tactile Vest for Astronauts in the International Space Station. Proc. Eurohaptics 2003, (2003).

[6] Wall, S. and Brewster, S. Feeling what you hear: tactile feedback for navigation of audio graphs. Proc, CHI 2006, ACM Press (2006) 